\title{
Performance Prediction of an OWC Wave Power Plant with 3-D Characteristics in Regular Waves
}

\author{
Do-Chun Hong*, † Keyyong Hong \\ * Division Center for Advanced Transportation Vehicles Chungnam National Univ.Daejon, Republic of Korea \\ + Maritime \& Ocean Engineering Research Institute, KIOST Daejon, Republic of Korea
}

\begin{abstract}
The primary wave energy conversion by a three-dimensional bottom-mounted oscillating water column (OWC) wave power device in regular waves has been studied. The linear potential boundary value problem has been solved following the boundary matching method. The optimum shape parameters such as the chamber length and the depth of the front skirt of the OWC chamber obtained through two-dimensional numerical tests in the frequency domain have been applied in the design of the present OWC chamber. Time-mean wave power converted by the OWC device and the time-mean second-order wave forces on the OWC chamber structure have been presented for different wave incidence angles in the frequency-domain. It has been shown that the peak period of $P_{m}$ for the optimum damping parameter coincides with the peak period of the time-mean wave drift force when $\gamma=0$.
\end{abstract}

Key words : three-dimensional wave diffraction theory, oscillating water column (OWC), bottom-mounted OWC wave power device, oscillating pressure drop, equivalent linear damping, frequency-domain wave power response amplitude operator

\section{Introduction}

The oscillating water column (OWC) wave power device consists of a partially submerged pneumatic chamber. The chamber encloses an oscillating water column separated from the outer surface waves by a front skirt while exposed to the outer wave field through an underwater opening below the skirt. The motion of the water column due to the incident waves produces a low pressure reciprocating air flow through a duct open to the atmosphere where a power take-off mechanism can be installed. In this paper, the primary wave energy conversion efficiency of a three-dimensional bottommounted OWC wave power device in regular waves has been studied. The theory of wave energy absorption by the OWC device has been presented by Evans (1982) as well as sarmento and Falcão (1985) for fixed devices and by Falnes and McIver (1985) for multiple floating devices. In this paper, the linear potential boundary value problem has been solved following the boundary matching method presented by Hong et al. (2004). The linear potential boundary value problem outside the OWC chamber has been formulated using the improved Green integral equation associated with the finite-water-depth free-surface Green function while the potential problem inside the OWC chamber using the Green integral equation associated with the fundamental Green function taking into account the oscillating air pressure on the free surface inside the chamber. The oscillating pressure drop in the pneumatic chamber above the OWC has been represented by a product of the mean air flow velocity through a duct in the air chamber and an equivalent linear damping which is given as a complex-valued parameter. Matching the inner and outer potentials as well as their normal derivatives on the common boundary, the whole potential boundary value problem has been solved. Numerical solution has been obtained via discretization using three-dimensional constant boundary element method. The magnitude and the peak frequency of the absorbed wave power depend significantly on shape parameters such as the chamber length and the depth of the front skirt of the OWC chamber. In order to clarify the effects of the shape parameters in view of the wave energy absorption efficiency, preliminary two-dimensional numerical tests were carried out by Hong et al. (2007). The results of the parametric tests have been used for the design of the present OWC chamber.

\section{Formulation of Potential Problem}

A bottom-mounted OWC chamber structure is located in finite depth water. Cartesian coordinates $(x, y, z)$ are

\footnotetext{
+ Corresponding author, khong@kiost.ac 042)866-3900

* dchong@cnu.ac.kr 042)866-3914
} 


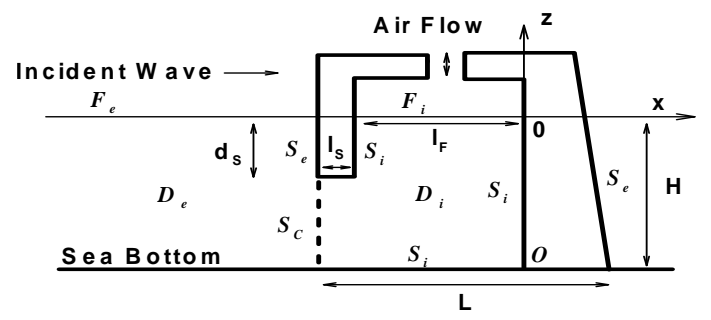

Fig. 1 Longitudinal section of a bottom-mounted OWC wave power device

employed with the origin $\mathrm{o}$ in the plane $z=0$ inside the chamber. A schematic view of the OWC chamber is shown in Fig. 1 where $l_{F}$ and $d_{S}$ denote the chamber length and the depth of the front skirt respectively. Assuming that the fluid is incompressible, the flow irrotational and the capillarity negligible, the complex-valued fluid velocity is given by the gradient of the complex-valued velocity potential as follows.

$$
v(x, y, z)=\nabla \Psi(x, y, z)
$$

The real-valued velocity potential can be obtained as follows.

$$
\Phi(x, y, z, t)=\operatorname{Re}\left\{\Psi(x, y, z) e^{-i \omega t}\right\}
$$

where $\omega$ is the circular frequency of plane progressive linear waves incident from the infinity. The complex-valued velocity potential $\Psi$ satisfies Laplace's equation as follows in the fluid region.

$$
\nabla^{2} \Psi=0
$$

Due to diffraction of waves by the structure, $\Psi$ is commonly expressed as the sum of the incident wave potential $\Psi_{0}$ and the scattering wave potential $\Psi_{S}$ as follows.

$$
\Psi=\Psi_{0}+\Psi_{S}
$$

The complex-valued incident wave potential is expressed as follows.

$$
\Psi_{0}=-\frac{\zeta_{0} \omega}{m_{0}} \frac{\cosh m_{0}(z+H)}{\sinh m_{0} H} e^{i m_{0}(x \cos \beta+y \sin \beta)}
$$

where $\zeta_{0}$ denotes the amplitude of the incident wave, $\beta$ the incident angle of waves relative to the positive $x$-axis, $H$ the water depth and $m_{0}$ the wave number satisfying the linear dispersion relation for finite depth waves defined as follows.

$$
m_{0} \tanh m_{0} H=k_{0}, k_{0}=\omega^{2} / g
$$

where $g$ is the gravitational acceleration. The complexvalued wave elevation $\zeta$ can be expressed as follows.

$$
\zeta=\zeta_{0} e^{i m_{0}(x \cos \beta+y \sin \beta)}
$$

The time-mean incident wave power per one meter width of wave-front can be obtained as follows.

$$
P_{0}=\frac{1}{2} \rho g C_{g} \zeta_{0}^{2}
$$

where $C_{g}$ is the group velocity defined as follows.

$$
C_{g}=\frac{\omega}{2 m_{0}}\left[1+\frac{2 m_{0} H}{\sinh \left(2 m_{0} H\right)}\right]
$$

Due to the wave diffraction, wave power devices cannot completely absorb the incident wave energy.

Here, we assume that pressure drop through the duct in the OWC chamber is linearized as follows.

$$
\operatorname{Re}\left[p_{C} e^{-i \omega t}\right]=\operatorname{Re}\left[\gamma U_{D} e^{-i \omega t}\right], \gamma=\mu+i \nu
$$

where $\gamma=\mu+i \nu$ denotes the complex-valued equivalent linear damping parameter, $U_{D}$ complex-valued mean velocity of the air flow through the duct and $p_{C}$

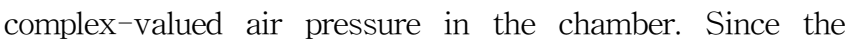
relative volume flow rate thorough the duct must be equal to that through $F_{i}$, the internal free surface at its mean position, we have

$$
U_{D}=\frac{1}{A_{D}} \iint_{F_{i}} \frac{\partial\left(\Psi_{S}+\Psi_{0}\right)}{\partial n} d s
$$

where $A_{D}$ denotes the sectional area of the duct.

Then the linearized free surface boundary condition can be found as follows.

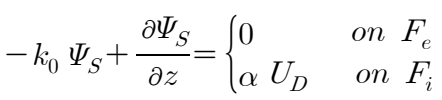

where $F_{e}$ denotes the outer free surface and $\alpha$ a non-dimensional linear damping parameter defined as follows.

$$
\alpha=i \gamma \omega / \rho g
$$

On the wetted surface $S$ of the body, $\Psi_{S}$ satisfies 


$$
\frac{\partial \Psi_{S}}{\partial n}=-\frac{\partial \Psi_{0}}{\partial n} \text { on } S
$$

and

$$
\frac{\partial \Psi_{S}}{\partial n}=0 \quad \text { on } z=-H
$$

The scattering potentials $\Psi_{S}$ must also satisfy the radiation condition at infinities. Here, the entire fluid region is divided into $D_{e}$, the region outside the chamber and $D_{i}$ inside by a control surface $S_{C}$ as shown in Fig. 1. Applying Green's theorem to the potential and the finite-water-depth free-surface Green function $G$, we have the following improved Green integral equation for the potential in $D_{e}$.

$$
\begin{aligned}
& \frac{\Psi_{S}(P)}{2}+\iint_{S_{e} \cup S_{C}} \Psi_{S}(M) \frac{\partial G(P, M)}{\partial n} d s \\
& -\iint_{S_{C}} \frac{\partial \Psi_{S}(M)}{\partial n} G(P, M) d s= \\
& -\iint_{S_{e}} \frac{\partial \Psi_{0}(M)}{\partial n} G(P, M) d s, \quad P \in S_{e} \cup S_{C} \cup S_{W}
\end{aligned}
$$

where $S_{W}$ is the waterplane of the chamber structure.

In $D_{i}$, applying Green's theorem to the potential and the fundamental Green function $G_{0}$, we have

$$
\begin{aligned}
& \frac{\Psi_{S}(P)}{2}-\iint_{S_{i} \cup S_{C}} \Psi_{S}(M) \frac{\partial G_{0}(P, M)}{\partial n} d s \\
& -\frac{1}{k_{0}} \iint_{F_{i}} \frac{\partial \Psi_{S}(M)}{\partial n} \frac{\partial G_{0}(P, M)}{\partial n} d s \\
& -\iint_{F_{i} \cup S_{c}} \frac{\partial \Psi_{0}(M)}{\partial n} G_{0}(P, M) d s= \\
& \iint_{S_{i}} \frac{\partial \Psi_{0}(M)}{\partial n} G_{0}(P, M) d s \\
& -\frac{1}{k_{0}} \alpha U_{D} \iint_{F_{i}} \frac{\partial G_{0}(P, M)}{\partial n} d s, \quad P \in S_{i} \cup S_{C} \cup F_{i}
\end{aligned}
$$

The boundary conditions on the matching boundary $S_{C}$ are as follows.

$$
\begin{aligned}
& \Psi_{S}\left(M^{+}\right)=\Psi_{S}(M \digamma) \\
& \frac{\partial \Psi_{S}\left(M^{+}\right)}{\partial n}=\frac{\partial \Psi_{S}(M)}{\partial n}, M \text { on } S_{C}
\end{aligned}
$$

where the superscripts + and - denote the inside and outside surfaces of $S_{C}$. Solving a system of integral equations obtained after combining the integral equation in the outer and inner regions with the aid of the matching boundary conditions on $S_{C}$, we can obtain solution as follows.

$$
\begin{aligned}
& \Psi_{S}=\phi-\frac{1}{k_{0}} \alpha U_{D} \alpha_{C} \text { on } S_{e} \cup S_{c} \cup S_{i} \\
& \frac{\partial \Psi_{S}}{\partial n}=\frac{\partial \phi}{\partial n}-\frac{1}{k_{0}} \alpha U_{D} \frac{\partial \phi_{C}}{\partial n} \text { on } F_{i} \cup S_{c}
\end{aligned}
$$

where $\Psi$ and $\Psi_{C}$ are solutions due to the first term and the integral of the second term of the right-hand side of (17) respectively. Using (11) and (20), the unknown $U_{D}$ can be determined.

\section{Time-mean wave power and wave loads}

The hydrodynamic pressure $p$ can be obtained by making use of the linearized Bernoulli equation.

$$
P=-\rho \frac{\partial \Phi}{\partial t}=\operatorname{Re}\left(p e^{-i \omega t}\right), \quad p=i \rho \omega \Psi
$$

The complex-valued air pressure $p_{C}$ in the chamber can be obtained using (10). The external forces and moments acting on the OWC chamber structure due to $p$ and $p_{C}$ can be obtained by integrating $p$ over the wetted surface and $p_{C}$ over the surface in contact with air respectively. The time-mean drift force $F_{d}$ can also be obtained using the near-field method presented by Pinkster and Oortmerssen (1997).

$$
F_{d}=-\frac{\rho g}{4} \oint_{W}|\eta|^{2} n d l+\frac{\rho}{2} \iint_{S_{e} \cup S_{i}} \frac{1}{2}|\nabla \Psi|^{2} n d s
$$

where $\eta$ denotes the wave elevation along the waterline of the chamber structure. The wave elevation inside the chamber can be obtained as follows.

$$
\eta=\frac{i \omega}{g} \Psi+i \frac{(\mu+i \nu)}{\omega} U_{D} \quad \text { in } F_{i}
$$

The time-mean value of the wave power converted by the OWC device $P_{m}$ can be obtained using a near-field formula as follows.

$$
P_{m}=\frac{1}{2} A_{D} \operatorname{Re} \gamma\left|U_{D}\right|^{2}
$$


Validation of (24) can be seen in the analogous two-dimensional numerical study where the absorbed wave power obtained using (24) has been shown to agree well with that obtained using the far-field formula (Hong and Hong, 2007). Since the time-mean incident wave power defined by (8) is for one meter width of wave-front, the three-dimensional primary wave power conversion efficiency Eff may be written as follows.

$$
E f f=P_{m} /\left(P_{0} \times b\right)
$$

where $b$ is the Inner width of the OWC chamber.

\section{Numerical results and discussions}

A series of computations for a bottom-mounted OWC chamber model I and II have been carried out successively. Numerical results for the model I have already been presented by Hong and Hong (2010). Discretized views of wetted surfaces of the model I and II are shown in Fig.2-3 respectively. Their main particulars are presented in Table 1. The water chamber of the model I is divided by 2 equivalent rooms. The chamber of the model II is not divided but stiffened with columns and beams as shown in Fig.3. Five different damping parameters - 0, 100+10i, $200+20 \mathrm{i}, 300+30 \mathrm{i}, 400+40 \mathrm{i}-$ have been used in the present computations for the model II. The time-mean wave drift forces acting on the OWC chamber structure of the model II for wave incidence angle $\beta=0,45$ and 90 degrees have been presented in Figs. 4-8. Figs. 9-11 show the time mean wave power $P_{m} / \zeta_{0}^{2}$ converted by the OWC model II for different damping parameters. They show that the optimum value of the damping parameter lies between 100+10i and 200+20i. This optimum value may be interpreted as a suitable physical factor for a secondary power take off mechanism.

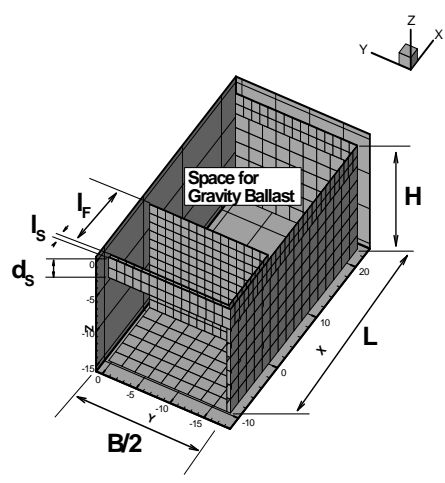

Fig. 2 Half view of OWC model I with Gravity Ballast Structure

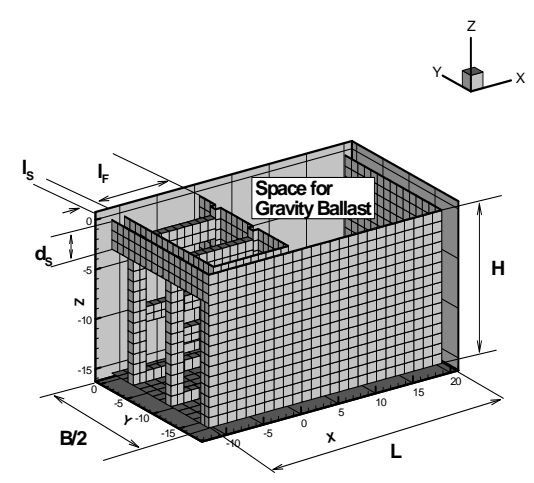

Fig. 3 Half view of OWC model II with gravity ballast structure

Table 1 Principal Particulars of OWC Chamber with Gravity Ballast Structure

\begin{tabular}{|c|c|c|}
\hline & Model I & Model II \\
\hline Water Depth & $H=15 \mathrm{~m}$ & $H=15.4 \mathrm{~m}$ \\
\hline Chamber Inner Width & $b=35 \mathrm{~m}$ & $b=35 \mathrm{~m}$ \\
\hline Chamber Length & $l_{F}=10 \mathrm{~m}$ & $l_{F}=10 \mathrm{~m}$ \\
\hline Front Skirt Draft & $d_{S}=3 \mathrm{~m}$ & $d_{S}=2.99 \mathrm{~m}$ \\
\hline Front Skirt Thickness & $l_{S}=0.5 \mathrm{~m}$ & $l_{S}=1.7 \mathrm{~m}$ \\
\hline Overall Width of the Structure & $B=36 \mathrm{~m}$ & $B=37 \mathrm{~m}$ \\
\hline Overall Length of the Structure & $L=32.5 \mathrm{~m}$ & $L=31.2 \mathrm{~m}$ \\
\hline
\end{tabular}

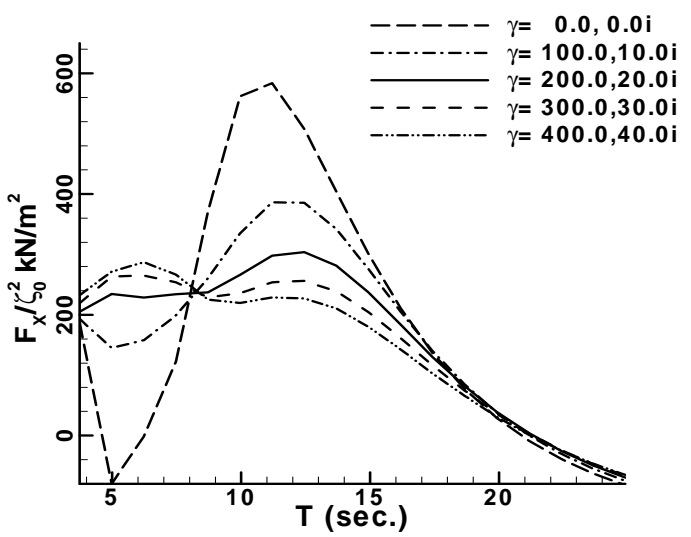

Fig. 4 Time-mean drift forces in $x^{-}$-direction, $\beta=0$ degs.

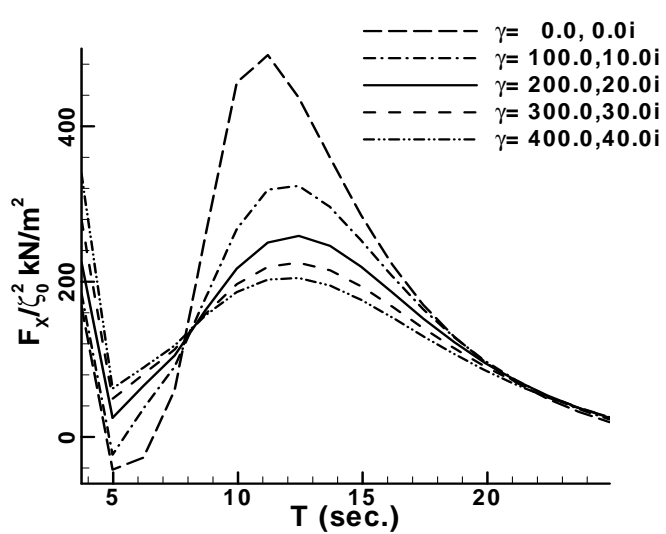

Fig. 5 Time-mean drift forces in $x^{-}$-direction, $\beta=45$ degs. 


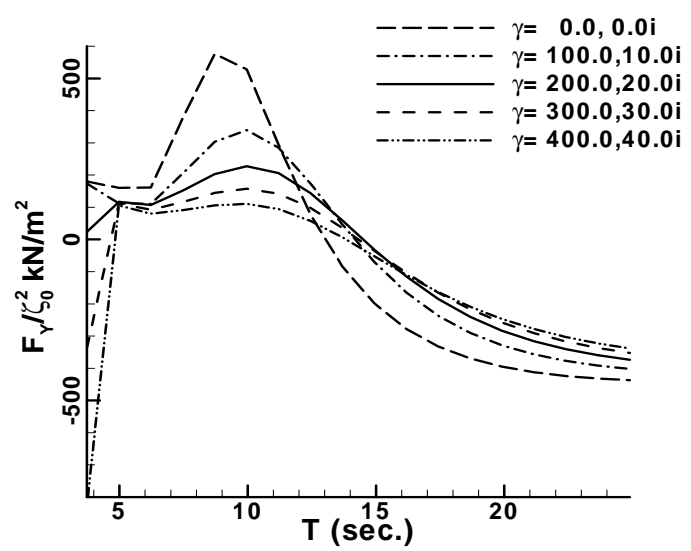

Fig. 6 Time-mean drift forces in $y^{-}$-direction, $\beta=45$ degs.

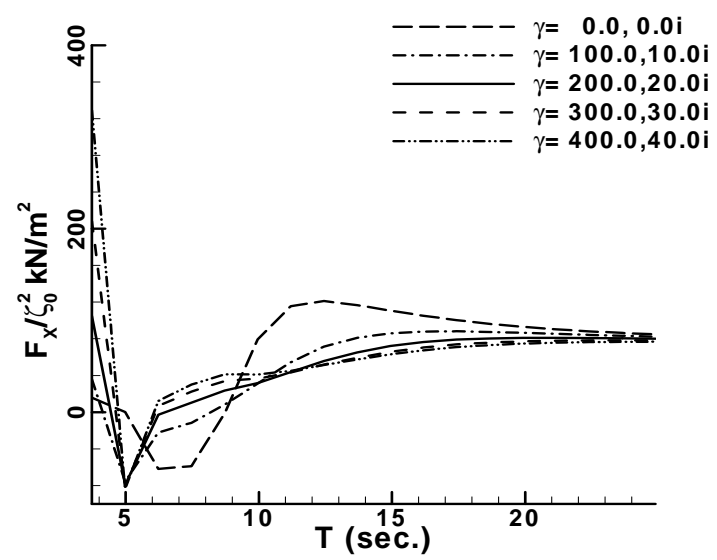

Fig. 7 Time-mean drift forces in $x$-direction, $\beta=90$ degs.

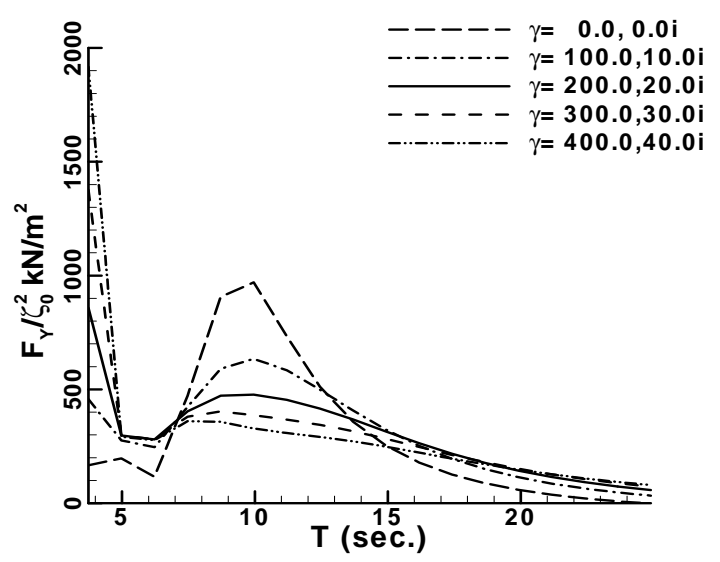

Fig. 8 Time-mean drift forces in $\mathrm{y}^{-}$-direction, $\beta=90$ degs.

Fig. 9 shows that the peak period of $P_{m}$ for $\beta=0 \mathrm{de}^{-}$ grees is about 11 seconds. This peak period of $\mathrm{Pm}$ for $\beta=0$ degrees coincides with the peak period of the time-mean wave drift force $F_{X}$ for $\beta=0$ degrees when $\gamma=0$ as shown in Fig. 4. Fig. 11 shows that the peak period of $P_{m}$ for $\beta$ $=90$ degrees is about 9 seconds. This peak period of

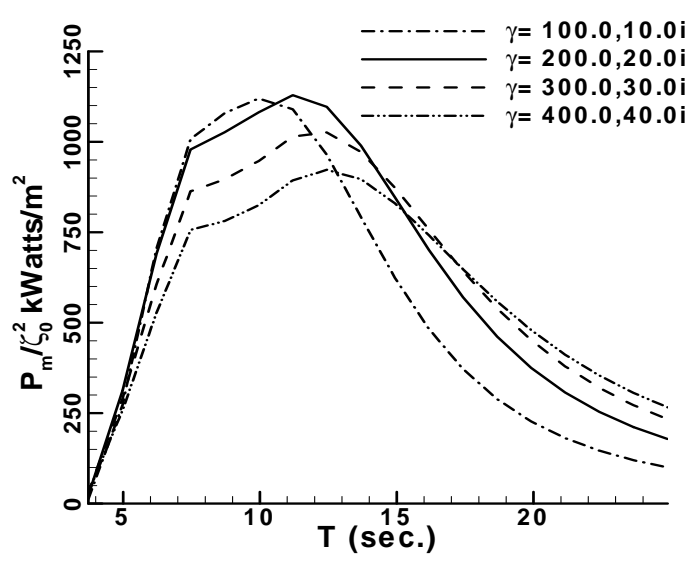

Fig. 9 Time-mean wave power converted by OWC device, $\beta=0$ degs.

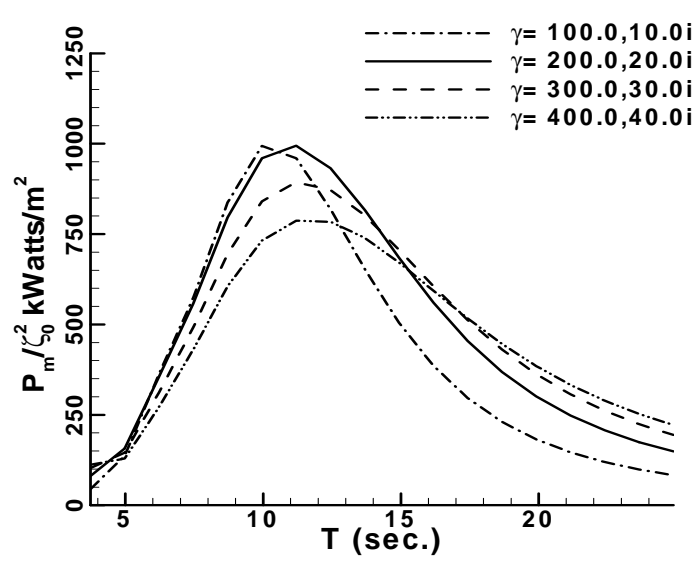

Fig. 10 Time-mean wave power converted by OWC device, $\beta=45$ degs.

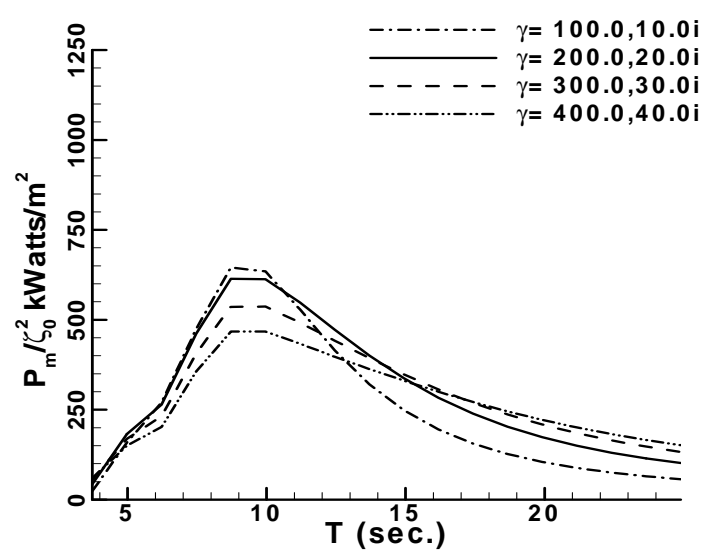

Fig. 11 Time-mean wave power converted by OWC device, $\beta=90$ degs

$P_{m}$ for $\beta=90$ degrees also coincides with the peak period of the time-mean wave drift force $F_{Y}$ for $\beta=90$ degrees when $\gamma=0$ as shown in Fig. 8. These facts suggest that the OWC chamber should be tuned to the peak period of 


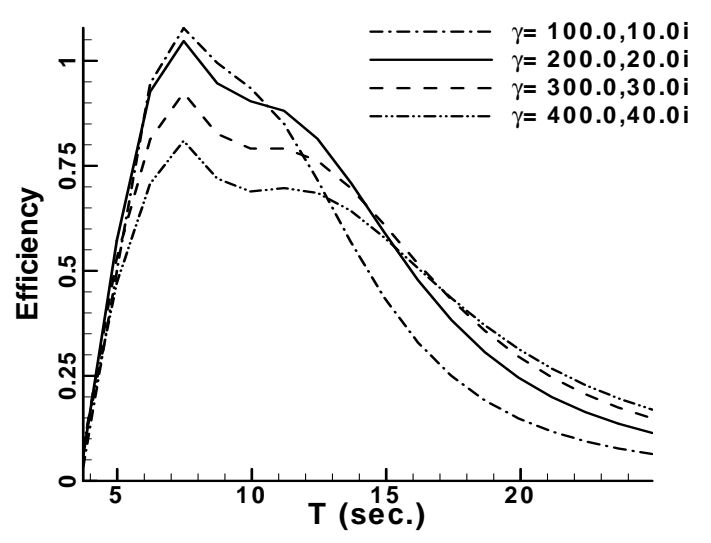

Fig. 12 Primary wave power conversion efficiencies of the model II for various damping parameters, $\beta=0$ degs.

the time-mean wave drift force to maximize conversion efficiency. Fig. 12 shows the primary wave power conversion efficiencies for various damping parameters for the model II. The peak periods of these curves are slightly below the peak periods of converted wave powers shown in Fig.9 since the wave power is proportional to the wave period. In Fig. 13, the primary wave power conversion efficiencies of the model I reported by Hong and Hong (2010) have been presented. Since main particulars of the model II differ slightly from the model I as shown in Table 1, they show similar behaviors. However, the model II, the present OWC chamber, show better efficiencies over a wide range of periods.

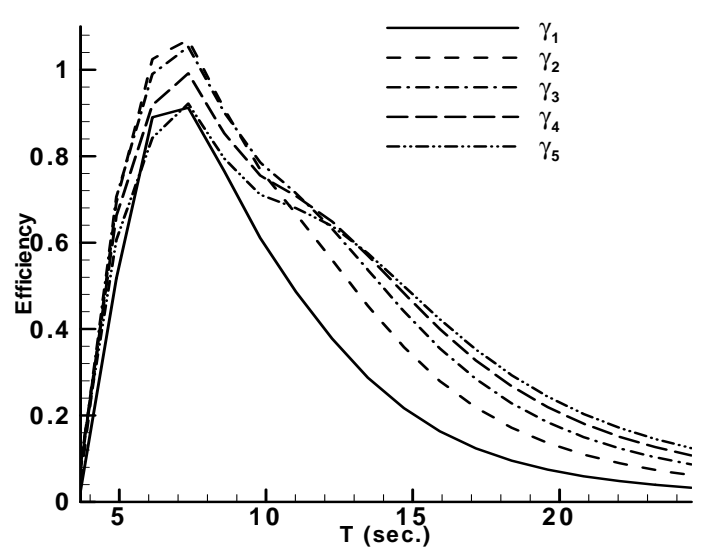

Fig. 13 Primary wave power conversion efficiencies of the model I for various damping parameters, $\beta=0$ degs., $\gamma_{1}=50+10 \mathrm{i}, \quad \gamma_{2}=100+20 \mathrm{i} \quad \gamma_{3}=150+30 \mathrm{i}, \quad \gamma_{4}=200+40 \mathrm{i}, \quad \gamma_{5}=$ $250+50 \mathrm{i}$

\section{Conclusion}

The time-mean wave power $P_{m}$ converted by a three-dimensional bottom-mounted OWC device using a linear damping parameter $\gamma$ and the time-mean second-order wave forces on the OWC chamber structure have been presented for different wave incidence angles in the frequency-domain. The optimum damping parameter has been found by comparing the wave power curves for various damping parameters. It has been shown that the peak period of $P_{m}$ for the optimum damping parameter coincides with the peak period of the time-mean wave drift force when $\gamma=0$. It suggests that the OWC chamber should be tuned to the peak period of the time-mean wave drift force when the OWC chamber is open to the atmosphere in order to maximize the primary wave power conversion efficiency. The curves of $P_{m} / \zeta_{0}^{2}$ can be used as the wave power response amplitude operators. Integrating the product of $P_{m} / \zeta_{0}^{2}$ and the wave energy spectrum in an irregular wave field over a frequency range, the convertible wave power in the wave field can be obtained.

\section{Acknowledgements}

The present work is a part of the research project on "Development of OWC wave energy utilization system" which is principally investigated by the Korea Institute of Ocean Science \& Technology and funded by the Ministry of Land, Transport and Maritime Affairs of Korea.

\section{References}

[1] Evans, D. V.(1982), "Wave-power absorption by systems of oscillating surface pressure distributions," J. Fluid Mech., Vol. 114, pp. 481-499.

[2] Falnes, J. and McIver, P.(1985), "Surface wave interactions with systems of oscillating bodies and pressure distributions," Applied Ocean Res. Vol. 7-4, pp. 225-234.

[3] Hong, D. C. and Hong, K. Y.(2010), "Prediction of wave energy absorption efficiency and wave loads of a three-dimensional bottom-mounted OWC wave power device," J. of the Korean Soc. for Marine Environmental Engr. Vol. 13-1, pp. 47-42.

[4] Hong, D. C. and Hong, S. Y.(2007), "Floating wave energy device with two oscillating water columns," Proc. 26th OMAE, paper No. OMAE2007-29103, Sandiego, USA.

[5] Hong, D. C., Hong, S. Y. and Hong, S. W.(2004), "Numerical study of the motions and drift force of a 
floating OWC device," Ocean Engineering, Vol. 31-2, pp. 139-164.4] Hong, D. C., Hong, S. Y. and Hong, S. W.(2004), "Numerical study of the motions and drift force of a floating OWC device," Ocean Engineering, Vol. 31-2, pp. 139-164.

[6] Hong, K. Y., Shin, S. H. and Hong, D. C.(2007), "Wave energy absorption efficiency of pneumatic chamber of OWC wave energy converter," J. of the Korean Soc. for Marine Environmental Engr. Vol. 10-3, pp. 173-180.

[7] Pinkster, J. A. and van Oortmerssen, G.(1977), "Computation of the first and second order wave forces on oscillating bodies in regular waves," Proc. 2nd Internat. Conf. on Numerical Ship Hydrodynamics, pp. 136-159.

[8] Sarmento, A.J.N.A. and Falcão, A. F. de O.(1985), "Wave generation by an oscillating surface-pressure and its application in wave-energy extraction ," J. Fluid Mech., Vol. 150, pp. 467-485.

Received 7 November 2012

Revised 26 November 2012

Accepted 26 November 2012 